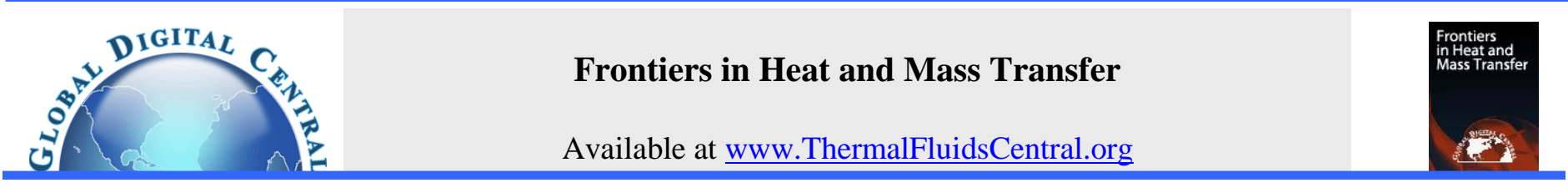

\title{
DUAL SOLUTIONS FOR HEAT AND MASS TRANSFER IN MHD JEFFREY FLUID IN THE PRESENCE OF HOMOGENEOUS- HETEROGENEOUS REACTIONS
}

\author{
C. S. K. Raju ${ }^{\mathrm{a}}$, N. Sandeep ${ }^{\mathrm{a}}$, J. Prakash ${ }^{\mathrm{b}, 1}$ \\ ${ }^{a}$ Fluid Dynamics Division, VIT University, Vellore-632014, India. \\ ${ }^{b}$ Department of Mathematics, University of Botswana, Gaborone, Botswana.
}

\begin{abstract}
In this study, we analyzed the effects of nonlinear thermal radiation and induced magnetic field on steady two-dimensional incompressible flow of Jeffrey fluid flow past a stretching/shrinking surface in the presence of homogeneous-heterogeneous reactions. For physical relevance in this study we analyzed the behavior of homogeneous and heterogeneous profiles individually. The transformed governing equations with the help of similarity variables are solved numerically via Runge-Kutta and Newton's method. We obtained better accuracy of the present results by differentiating with the existed published literature. The effect of pertinent parameters on velocity, induced magnetic field, temperature and concentration profiles along with the friction factor and local Nusselt number are presented with the help of graphs and tables. Results imply that the Deborah number have tendency to increase the heat transfer rate. Concentration profiles are positively influenced by the homogeneous-heterogeneous reactions, which assist to enlarge the concentration profiles.
\end{abstract}

Keywords: Induced magnetic field, Homogeneous-heterogeneous reactions, nonlinear thermal radiation, Jeffrey fluid, stretching/shrinking sheet.

\section{INTRODUCTION}

The study of boundary layer flows over a stretching surface has enlarged various applications in pharmaceutical companies (i.e. medicinal pharmaceutical technology), polythene, polymer industries, preparation of elastic sheets, paper production, optical fiber cable manufacturing, and manufacturing of plastic, science and engineering technology. Physically, the pattern of thermal radiation which occurs during some of the industrial processes may not be interpreted as linear. To fill this gap, in this study we consider the thermal radiation as nonlinear. In industries, almost all the fluids may be accurately referred to as material that exhibits both viscous and elastic characteristics when undergoing deformation. Keeping this into view, Abbas et al. (2015) illustrated the slip condition effects on stagnationpoint flow of a MHD viscous fluid flow past a stretching/shrinking surface and concluded that the velocity profiles are decreases with an increase in the shrinking parameter. Abbasi et al. (2015) discussed the MHD Jeffrey nanofluid flow past a stretching surface with mass flux conditions and concluded that the thermal boundary layer thickness enhanced with the Brownian motion parameter.

The effect of induced magnetic field on stagnation point flow towards a stretching surface was analyzed by Ali et al. (2011). Thermodiffusion influence on an axisymmetric flow of a Jeffrey fluid past a stretching sheet was investigated by Awais et al. (2015). Chaudhary and Merkin (1995) depicted the effect of homogeneous-heterogeneous reactions on boundary layer flows. Dual solutions of an unsteady heat transfer analysis on an accelerating sheet in the presence of suction or blowing effects was discussed by Cheng and Huang (2004). Dabiri and Tryggvason (2015) examined the convection flow over a vertical channel. An analytical solution for three dimensional flow of Jeffrey fluid towards a stretching surface was discussed by Hayat et al. (2012). The heat source effect on Jeffrey fluid flow over a permeable stretching sheet was analyzed by Hayat et al. (2015). In this study they concluded that the velocity profiles are enhanced for higher values of porosity parameter. MHD second grade fluid flow past a shrinking sheet was analytically studied by Hayat et al. (2007). Boundary layer analysis of convective stagnation point flow through a vertical stretching surface was investigated by Ishak et al. (2006). Chemically reacting magneto hydrodynamic flow over a vertical cone was investigated by Mallikarjuna et al. (2015). Mahapatra and Guptha (2002) illustrated the heat transfer characteristics of stagnation-point flow through a stretching surface. Mohankrishna et al. (2015) investigated the influence of thermal radiation and suction on free convection flow past a permeable stretching sheet.

An unsteady heat transfer analysis of a nanofluid over a shrinking sheet in the presence of suction effect was considered by Rohni et al. (2012). Nazar et al. (2004) depicted an unsteady heat transfer analysis of stagnation-point flow through a stretching surface. Qasim (2013) considered the heat transfer analysis of Jeffery fluid flow past a stretching surface. Ramana Reddy et al. (2015) considered the magneto hydrodynamic nanofluid flow past a permeable vertical moving plate in the presence of thermal radiation and soret effect. A thermal radiation influence on MHD ferrofluid flow into a flat plate in the presence of non-uniform heat source/sink was examined by Sandeep et al. (2015). Sheikhoslami and Abelman (2015) studied the heat transfer characteristics of MHD nanofluid flow past a horizontal circular cylinder. Raju et al. (2015) considered the thermal radiation influence on MHD flow past a stretching sheet in the presence of aligned magnetic field. Turkyilmazoglu and Pop (2013) analyzed the stagnation point flow of Jeffrey fluid towards a stretching surface. Two-dimensional micro-polar fluid flow past a shrinking surface was depicted by Yacob and Ishak (2012). Recently, Sulochana and Sandeep $(2015,2016)$ analyzed heat transfer characteristics on MHD flows.

In view of all the citations above mentioned are mostly related to stretching sheet. In this study a little attention has been given to investigate the motion of Jeffrey fluid flow towards a stretching/shrinking surface in the presence of nonlinear thermal radiation. In addition, no attempt has been made to investigate the behavior of Jeffrey fluid choosing the influence of nonlinear thermal 
radiation, induced magnetic field, magnetic diffusivity of the fluid and homogeneous-heterogeneous reaction which occurs in the fluid as it flows. The transformed governing equations are solved numerically via Runge-Kutta and Newton's method. The influence of dimensionless parameters on velocity, induced magnetic field, temperature and concentration profiles along with the friction factor and local Nusselt number are discussed with the help of graphs and tables.

\section{MATHEMATICAL FORMULATION}

In this study we consider a steady, incompressible, electrically conducting Jeffrey fluid past a permeable stretching/shrinking sheet in the presence of induced magnetic field and homogeneousheterogeneous reactions. The stretching sheet is considered along the $x$ - axis. The $y$-axis is normal to it. It is assumed that the applied magnetic field is of strength $B_{0}$. It is also assumed that an induced magnetic field is applied in $y$-direction and the parallel component of induced magnetic field $B_{1}$ approaches the value $B_{e}=B_{0}$ in the free stream flow, where $B_{e}$ is the magnetic field strength at free stream as displayed in Fig.1. It is considered that $u_{w}=c x$ and $u_{e}=d x$ are the stretching and free stream velocities respectively, where $c, d$ are constants. It is assumed a simple homogeneous-heterogeneous model as per Chaudhary and Merkin (1995) in the following form:

$$
A+2 B \rightarrow 3 B, \text { rate }=k_{c} a b^{2},
$$

while on the catalyst surface we have the single, isothermal first order chemical reaction

$$
A \rightarrow B, \text { rate }=k_{s} a,
$$

where $a$ and $b$ are the concentration of the chemical species $A, B$ respectively and $k_{s}, k_{c}$ are rate constants.

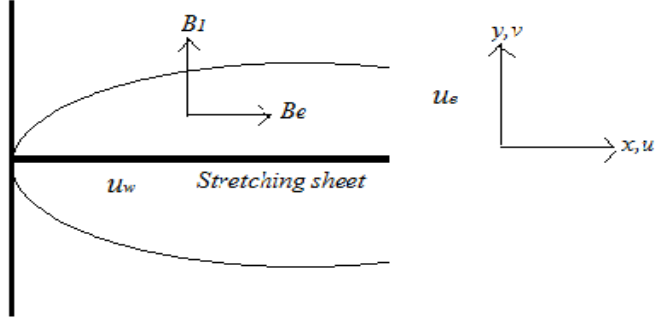

Fig. 1 Physical model of the problem

Under the above assumptions the governing boundary layer equations are given by:

\section{Continuity equations:}

$$
\begin{aligned}
& \frac{\partial u}{\partial x}+\frac{\partial v}{\partial y}=0 \\
& \frac{\partial B_{1}}{\partial x}+\frac{\partial B_{2}}{\partial y}=0
\end{aligned}
$$

Momentum equation:

$$
\begin{aligned}
& \rho\left(u \frac{\partial u}{\partial x}+v \frac{\partial u}{\partial y}\right) \\
& =\left(u_{e}(x) \frac{\partial u_{e}(x)}{\partial x}-\frac{\mu}{4 \pi} B_{e} \frac{\partial B_{e}}{\partial x}\right)+\frac{\mu}{4 \pi}\left(B_{1} \frac{\partial B_{1}}{\partial x}+B_{2} \frac{\partial B_{1}}{\partial y}-B_{e} \frac{\partial B_{e}}{\partial x}\right) \\
& +\frac{v}{1+\lambda_{1}}\left[\frac{\partial^{2} u}{\partial y^{2}}+\lambda_{2}\left(\frac{\partial u}{\partial y} \frac{\partial^{2} u}{\partial x \partial y}+u \frac{\partial^{3} u}{\partial x \partial y^{2}}-\frac{\partial u}{\partial x} \frac{\partial^{2} u}{\partial y^{2}}+v \frac{\partial^{3} u}{\partial y^{3}}\right)\right]-\frac{v}{k} u
\end{aligned}
$$

\section{Induced magnetic field equation:}

$$
u \frac{\partial B_{1}}{\partial x}+v \frac{\partial B_{1}}{\partial y}=B_{1} \frac{\partial u}{\partial x}+B_{2} \frac{\partial u}{\partial y}+\mu_{e} \frac{\partial^{2} B_{1}}{\partial y^{2}},
$$

Energy equation:

$$
\left(u \frac{\partial T}{\partial x}+v \frac{\partial T}{\partial y}\right)=\alpha \frac{\partial^{2} T}{\partial y^{2}}+\frac{16 \sigma^{*}}{3 k^{*} \rho c_{p}} \frac{\partial}{\partial y}\left(T^{3} \frac{\partial T}{\partial y}\right),
$$

Diffusion equation with homogeneous-heterogeneous reactions:

$$
\begin{aligned}
& \left(u \frac{\partial a}{\partial x}+v \frac{\partial a}{\partial y}\right)=D_{A} \frac{\partial^{2} a}{\partial y^{2}}-k_{c} a b^{2}, \\
& \left(u \frac{\partial b}{\partial x}+v \frac{\partial b}{\partial y}\right)=D_{B} \frac{\partial^{2} b}{\partial y^{2}}+k_{c} a b^{2},
\end{aligned}
$$

subject to the boundary conditions

$$
\begin{aligned}
& u=u_{w}(x)=\lambda c x, v=v_{w}(x), \frac{\partial B_{1}}{\partial y}=B_{2}=0, \\
& T=T_{w}, D_{A} \frac{\partial a}{\partial y}=k_{s} a(y), D_{B} \frac{\partial b}{\partial y}=-k_{s} a(y) \quad \text { at } y=0, \\
& u=u_{e}(x)=\lambda a x, \frac{\partial u}{\partial y} \rightarrow 0, B_{1}=B_{e}(x)=B_{0} x, \\
& T=T_{\infty}, a(y)=a_{0}, b(y)=0 \text { as } y \rightarrow \infty,
\end{aligned}
$$

$\mu_{e}$ is the magnetic diffusivity of the fluid, which is given by

$$
\mu_{e}=\frac{1}{4 \pi \sigma},
$$

To convert the governing equations into set of nonlinear ordinary differential equations, we now introduce the following similarity transformation.

$$
\begin{aligned}
& u=c x f^{\prime}(\eta), v=-v_{f}^{0.5} c^{0.5} f(\eta), \eta=v_{f}{ }^{-0.5} c^{0.5} y, \\
& G(\eta)=a / a_{0}, H(\eta)=b / a_{0}, \\
& B_{1}=B_{0} x g^{\prime}(\eta), B_{2}=-B_{0} v_{f}^{0.5} c^{-0.5} g(\eta) \text {, } \\
& \theta(\eta)=\left(T-T_{\infty}\right) /\left(T_{w}-T_{\infty}\right) \text { or } \mathrm{T}=\mathrm{T}_{\infty}\left(1+\left(\theta_{w}-1\right) \theta\right),
\end{aligned}
$$

Substituting equations (10)-(12) in to (3)-(9), then equations (3) and (4) will be satisfied continuity equation automatically. Now, the equations (5)-(9) will be transformed into the following nonlinear coupled ordinary differential equations:

$$
\begin{gathered}
f^{\prime \prime \prime}-\left(1+\lambda_{1}\right)\left(f^{\prime 2}-f f f^{\prime \prime}\right)+M\left(g^{\prime 2}-g g^{\prime \prime}-1\right)+ \\
(d / c)^{2}+\beta\left(f^{\prime \prime 2}-f f^{\prime \prime \prime}\right)-\Lambda f^{\prime}=0, \\
\varepsilon g^{\prime \prime \prime}+f g^{\prime \prime}-f^{\prime \prime} g=0, \\
\theta^{\prime \prime}\left(1+R\left(1+\left(\theta_{w}-1\right) \theta\right)^{3}\right)+\operatorname{Pr} f \theta^{\prime} \\
+\left(3\left(\theta_{w}-1\right) \theta^{\prime 2}\left(1+\left(\theta_{w}-1\right) \theta\right)^{2}\right)=0, \\
G^{\prime \prime}+S c\left(f G^{\prime}-K G H^{2}\right)=0, \\
\delta H^{\prime \prime}+S c\left(f H^{\prime}+K G H^{2}\right)=0,
\end{gathered}
$$

with the transformed boundary conditions

$$
\begin{aligned}
& f=S, g=0, f^{\prime}=\lambda, g^{\prime \prime}=0, \theta=1, \\
& G^{\prime}=K_{s} G, \delta H^{\prime}=-K_{s} G \quad \text { at } \eta=0 \text {, } \\
& f^{\prime}=d / c, g^{\prime}=1, f^{\prime \prime}=0, \theta=0 \text {, } \\
& G=1, H=0 \\
& \text { as } \eta \rightarrow \infty \text {, }
\end{aligned}
$$

where $\beta$ is the Deborah number, $M$ is the magnetic parameter, $\varepsilon$ is the reciprocal magnetic Prandtl number, Pr the Prandtl number, $S c$ is the Schmidt number, $\Lambda$ is the porosity parameter, $K$ is the measure of strength of homogeneous reaction, $K_{s}$ is the strength of heterogeneous reaction, $\mathrm{Re}=c / v$ is the Reynolds number, $S$ is the 
suction parameter, $\lambda$ is the stretching/shrinking parameter, with $\lambda>0$ for stretching sheet and $\lambda<0$ for shrinking case and $\delta=D_{A} / D_{B}$ is the ratio of diffusion coefficient, which are represented below.

$$
\left.\begin{array}{l}
M=\frac{\mu H_{0}^{2}}{4 \pi \rho c^{2}}, \mathrm{~K}=\frac{v}{k c}, \varepsilon=\frac{1}{4 \pi \sigma v}, \beta=\lambda_{2} c, R=\frac{16 \sigma^{*} T_{\infty}^{3}}{3 k k^{*}}, \\
\operatorname{Pr}=\frac{v}{\alpha}, S c=\frac{v}{D_{A}}, K=\frac{k_{c} a_{0}^{2}}{c}, K_{s}=\frac{k_{s}}{D_{A} \sqrt{\operatorname{Re}}}, S=\frac{v_{w}}{\sqrt{v c}}
\end{array}\right\}
$$

In most of the applications diffusion coefficient $D_{A}$ and $D_{B}$ are making as equal by considering $\delta=1$. In this study we have made an attempt to show the individual behavior of the homogeneousheterogeneous profiles.

For physical quantities of engineering interest are the shear stress coefficient $C_{f}$ (friction factor) and the local Nusselt number $N u_{x}$ are given by

$$
\left.C_{f}=\frac{\tau_{w}}{\rho_{f} U_{w}^{2}}, \quad N u_{x}=\frac{x q_{w}}{k_{f}\left(T_{w}-T_{\infty}\right)},\right\}
$$

Here, $\tau_{w}$ is the wall shear stress and $q_{w}$ is the wall heat flux, which are given by

$$
\left.\tau_{w}=\mu\left(\frac{\partial u}{\partial y}\right)_{y=0}, q_{w}=-k\left(\frac{\partial T}{\partial y}\right)_{y=0},\right\}
$$

Using equations (12) in (20) and (21), we get

$$
\begin{aligned}
& \operatorname{Re}_{x}^{1 / 2} C_{f}=\left(\frac{1-\beta}{1+\lambda_{1}}\right) f^{\prime \prime}(0), \\
& \operatorname{Re}_{x}^{-1 / 2} N u_{x}=-\theta^{\prime}(0),
\end{aligned}
$$

where $\operatorname{Re}_{x}=u_{w} x / v$ is the local Reynolds number.

\section{RESULTS AND DISCUSSION}

The nonlinear ordinary differential equations (13) - (17) subjected to the boundary conditions (18) are solved numerically using RungeKutta and Newton's method proposed by Mallikarjuna et al. (2015). The effect of various non-dimensional governing parameters on velocity, induced magnetic field, temperature and concentration profiles along with the friction factor coefficient and local Nusselt number are discussed. For numerical calculations we considered $\varepsilon=0.1, R=1, \Lambda=0.5, d / c=0.2, \theta_{w}=1.1, \operatorname{Pr}=2, \mathrm{Sc}=0.6$,

$\delta=2, K=K_{s}=M=S=\lambda_{1}=\beta=1$. These values are remaining as constant in entire study except the varied values in the corresponding graphs and tables.

Figs. 2-6 display the variations of the velocity, temperature and concentration profiles for stretching/shrinking cases due to the change in the suction parameter. It is observed that an increase in the suction parameter depreciates the momentum and induced magnetic boundary layers over a shrinking surface. But we have seen an opposite results in stretching case. It is also noticed a fall in $H(\eta)$ profiles along with the temperature profiles due to an increase in the suction parameter. But homogeneous concentration profiles show reverse action to that of $H(\eta)$ profiles. Basically, higher values of suction parameter cause to decrease the thermal boundary layer thickness. Due to this reason we have seen a mixed response in the concentration profiles.

In order to depict the effect of radiation parameter on temperature profiles are plotted in Fig 7. We observed the result as we expected. That is an increasing in the thermal radiation parameter improves the thermal boundary layer thickness for both stretching/shrinking cases. It maybe happens due to the generation of a high thermal motion in the flow. Figs. 8-12 show the effect of magnetic field parameter on velocity, temperature, induced magnetic field and concentration profiles for both stretching/shrinking cases. It can be noted that for higher values of the magnetic parameter as in the case of shrinking surface the velocity, induced magnetic field and homogeneous concentration profiles are enhanced with the magnetic field parameter, whereas the thermal boundary layer thickness along with the heterogeneous concentration is reduced with an increase in the magnetic field parameter in shrinking case. The quite opposite results have been observed with an increase in the magnetic field parameter for stretching case.

Figs. 13-17 illustrate the influence of the Deborah number on velocity, induced magnetic field and concentration profiles for both stretching/shrinking cases. It observed a reverse type of results for both stretching and shrinking cases as we observed in magnetic field case. The variations of the strength of heterogeneous reaction parameter on concentration profiles are plotted in Figs. 18 and 19. It is evident that with an increase in $K_{s}$, we have seen depreciation in the $G(\eta)$ profiles and a raise in the $H(\eta)$ profiles. This may happen due to the variation in the strength of homogeneous reaction causes to fluctuate the diffusivity of the flow.

Table 1 Comparison of the skin friction coefficient and local Nusselt number when $K=K_{s}=S c=\beta=\theta_{w}=\lambda_{1}=R=\Lambda=0$.

\begin{tabular}{|c|c|c|c|c|}
\hline \multirow{2}{*}{$M$} & \multicolumn{4}{|c|}{$d / c=3$} \\
\cline { 2 - 5 }$M$ & $\begin{array}{c}C_{f} \\
\text { Ali et al. } \\
(2011)\end{array}$ & $\begin{array}{c}N u_{x} \\
\text { Ali et al. } \\
(2011)\end{array}$ & $\begin{array}{c}C_{f} \\
\text { Present } \\
\text { Results }\end{array}$ & $\begin{array}{c}N u_{x} \\
\text { Present } \\
\text { Results }\end{array}$ \\
\hline 0.1 & 4.70928 & 0.97902 & 4.70927 & 0.97902 \\
\hline 0.5 & 4.62764 & 0.97617 & 4.62765 & 0.97617 \\
\hline 1 & 4.52158 & 0.97240 & 4.52158 & 0.97240 \\
\hline & \multicolumn{4}{|c|}{$d / c=0.5$} \\
\cline { 2 - 5 }$M$ & $C_{f}$ & $N u_{x}$ & $C_{f}$ & $N u_{x}$ \\
& Ali et al. & Ali et al. & Present & Present \\
& $(2011)$ & $(2011)$ & Results & Results \\
\hline 0.1 & -0.57595 & 0.59171 & -0.57595 & 0.59171 \\
\hline 0.15 & -0.50938 & 0.60207 & -0.50938 & 0.60207 \\
\hline 0.2 & -0.40717 & 0.61811 & -0.40717 & 0.61811 \\
\hline
\end{tabular}

Table 2 Comparison of the friction factor when $K=K_{s}=S c=M=\beta=\theta_{w}=\lambda_{1}=R=\Lambda=0$.

\begin{tabular}{|c|c|c|c|}
\hline$d / c$ & $\begin{array}{c}\text { Ishak et al. } \\
(2006)\end{array}$ & $\begin{array}{c}\text { Nazar et al. } \\
(2004)\end{array}$ & $\begin{array}{c}\text { Present } \\
\text { Results }\end{array}$ \\
\hline 0.1 & -0.9694 & -0.9694 & -0.96941 \\
\hline 0.2 & -0.9181 & -0.9181 & -0.91810 \\
\hline 0.5 & -0.6673 & -0.6673 & -0.66731 \\
\hline 2 & 2.0175 & 2.0176 & 2.01750 \\
\hline 3 & 4.7294 & 4.7296 & 4.72931 \\
\hline
\end{tabular}

Tables 1 and 2 illustrate the validation of the present results with already published literature. We found an excellent agreement of the present results with the existed results. This proves the validity of the present results along with the accuracy of the numerical technique we used in this study. Table 3 depicts the effect of various physical parameters on skin friction coefficient and local Nusselt number. It is noted from the table that skin friction coefficient and local Nusselt number is improved with the Deborah number in case of stretching case. The quite opposite results have been observed in shrinking case. We have seen reverse results to above with an increase in the magnetic field parameter. Physically, rising values of magnetic field parameter enhance the drag force. This leads to reduce the wall friction. It is also 
observed that an increase in the nonlinear thermal radiation parameter depreciates the heat transfer rate for both stretching and shrinking cases. Generally, increasing values of radiation parameter develops the heat energy in the flow, and these causes enhance the temperature field and thus reduce the heat transfer rate. But an increase in the suction parameter shows opposite results to above. The strength of heterogeneous parameter does not shown significant influence on friction factor and heat transfer rate. Physically, homogeneousheterogeneous reactions are highly significant in controlling the mass transfer rate.

Table 3 Variation in physical parameter values

\begin{tabular}{|c|c|c|c|c|c|c|c|}
\hline$\lambda$ & $\beta$ & $M$ & $K_{s}$ & $R$ & $S$ & $f^{\prime \prime}(0)$ & $-\theta^{\prime}(0)$ \\
\hline 1 & 1 & & & & & -0.727272 & 1.236121 \\
\hline & 1.5 & & & & & -0.672452 & 1.244869 \\
\hline & 2 & & & & & -0.633151 & 1.250746 \\
\hline-1 & 1 & & & & & 2.473133 & 0.703764 \\
\hline & 1.5 & & & & & 2.176085 & 0.673812 \\
\hline & 2 & & & & & 2.010554 & 0.653420 \\
\hline 1 & & 1 & & & & -0.735229 & 1.235233 \\
\hline & & 3 & & & & -1.283583 & 1.189053 \\
\hline & & 6 & & & & -1.656889 & 1.164745 \\
\hline-1 & & 1 & & & & 2.470072 & 0.705659 \\
\hline & & 3 & & & & 3.036946 & 0.758110 \\
\hline & & 6 & & & & 3.494584 & 0.787332 \\
\hline 1 & & & 0.5 & & & -0.735229 & 1.235233 \\
\hline & & & 1 & & & -0.735229 & 1.235233 \\
\hline & & & 1.5 & & & -0.735229 & 1.235233 \\
\hline-1 & & & 0.5 & & & 2.470072 & 0.705659 \\
\hline & & & 1 & & & 2.470072 & 0.705659 \\
\hline & & & 1.5 & & & 2.470072 & 0.705659 \\
\hline 1 & & & & 0.5 & & -0.735229 & 1.640858 \\
\hline & & & & 1 & & -0.735229 & 1.235233 \\
\hline & & & & 1.5 & & -0.735229 & 1.001773 \\
\hline-1 & & & & 0.5 & & 2.470072 & 0.961730 \\
\hline & & & & 1 & & 2.470072 & 0.705659 \\
\hline & & & & 1.5 & & 2.470072 & 0.569655 \\
\hline 1 & & & & & 2 & -0.228853 & 2.032589 \\
\hline & & & & & 2.2 & -0.160288 & 2.192404 \\
\hline & & & & & 2.4 & -0.095260 & 2.352830 \\
\hline-1 & & & & & 2 & 1.169399 & 1.417662 \\
\hline & & & & & 2.2 & 1.037362 & 1.585072 \\
\hline & & & & & 2.4 & 0.924606 & 1.757287 \\
\hline & & & & & & \\
\hline
\end{tabular}

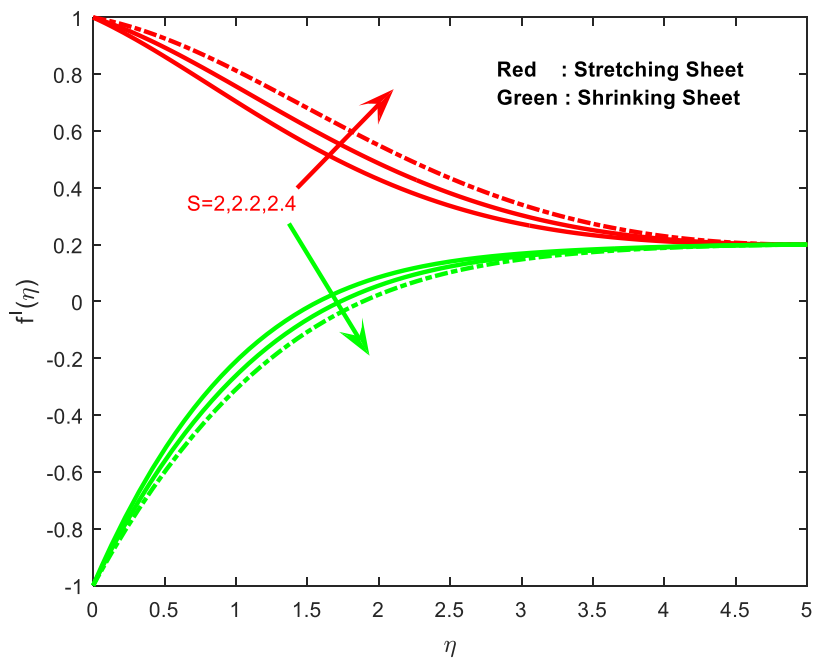

Fig. 2 Variations of $f^{\prime}(\eta)$ for different values of suction parameter

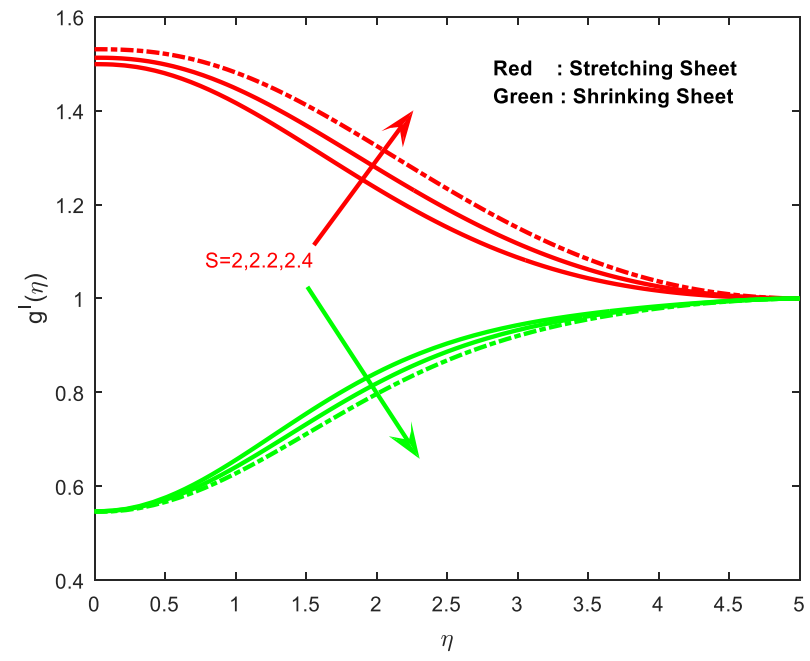

Fig. 3 Variations of $g^{\prime}(\eta)$ for different values of suction parameter

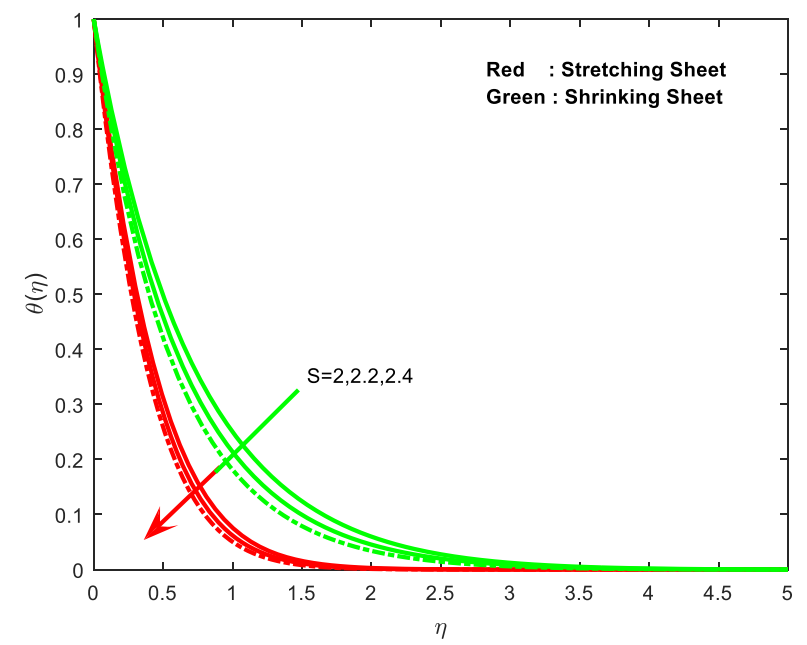

Fig. 4 Temperature profiles for different values of suction parameter

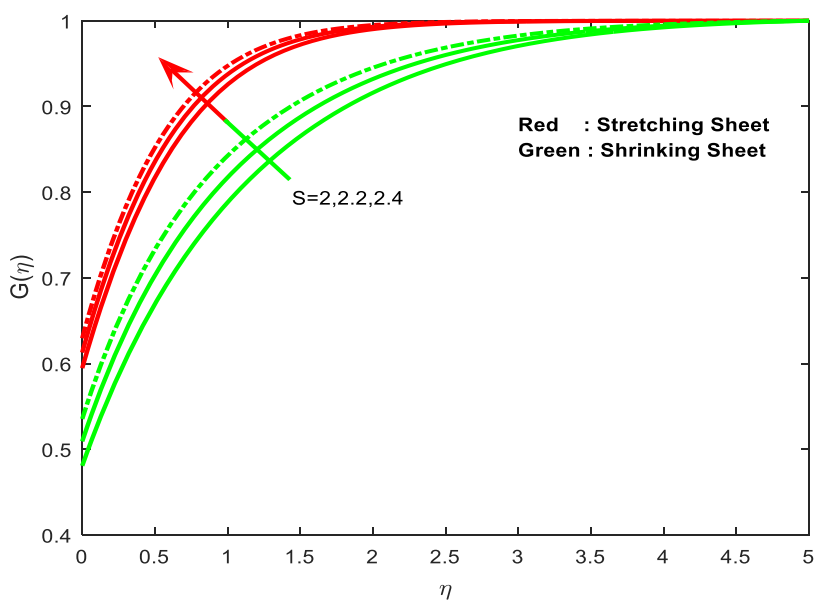

Fig. 5 Variations of $G(\eta)$ for different values of suction parameter 


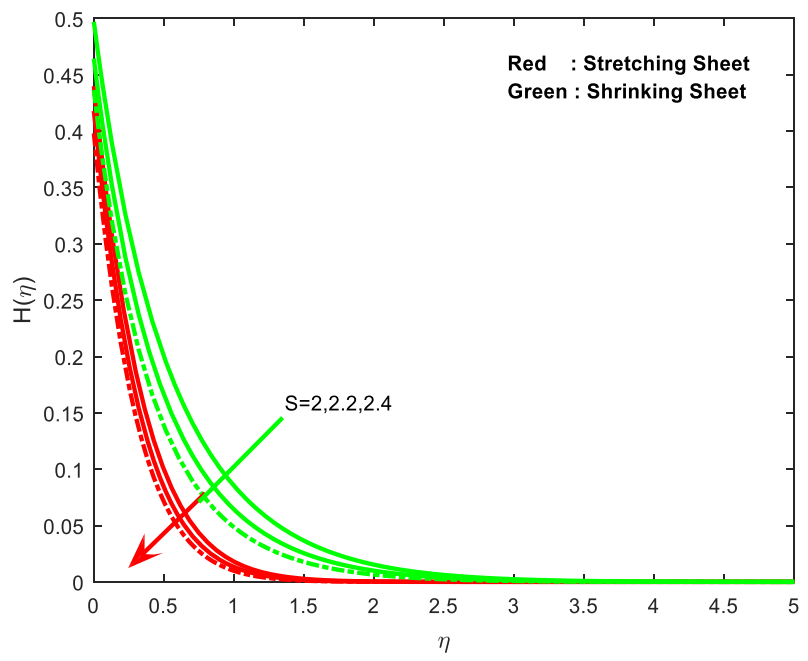

Fig. 6 Variations of $H(\eta)$ for different values of suction parameter

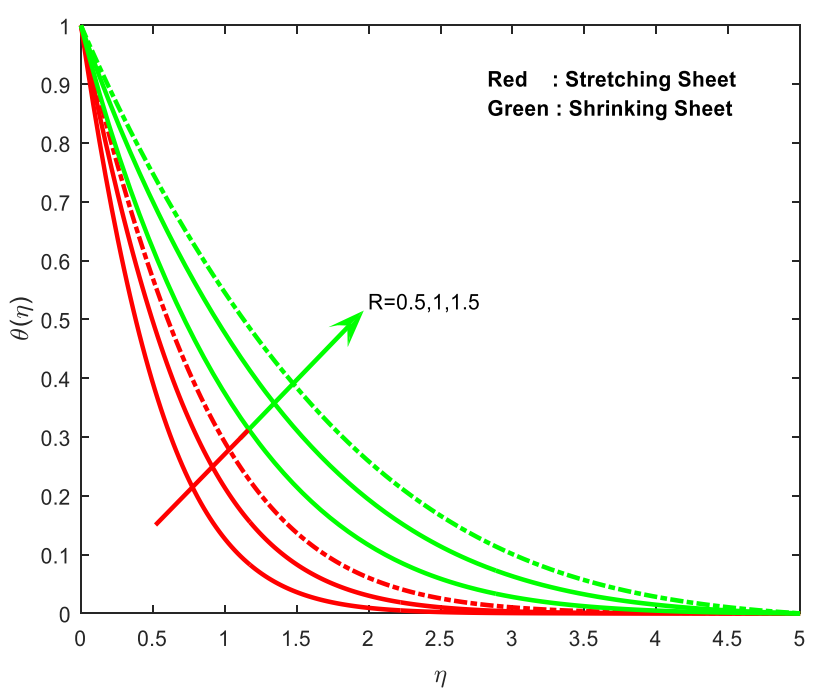

Fig. 7 Temperature profiles for different values of thermal radiation parameter

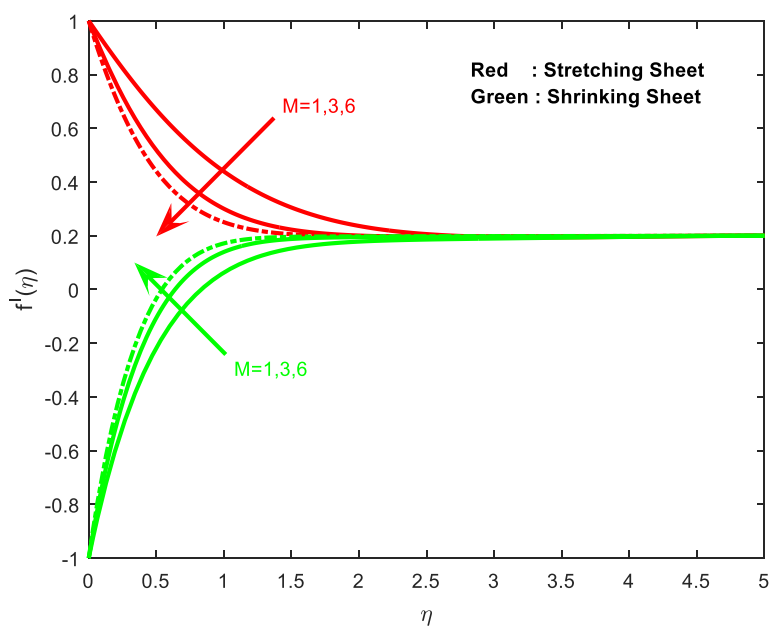

Fig. 8 Variations of $f^{\prime}(\eta)$ for different values of magnetic field parameter

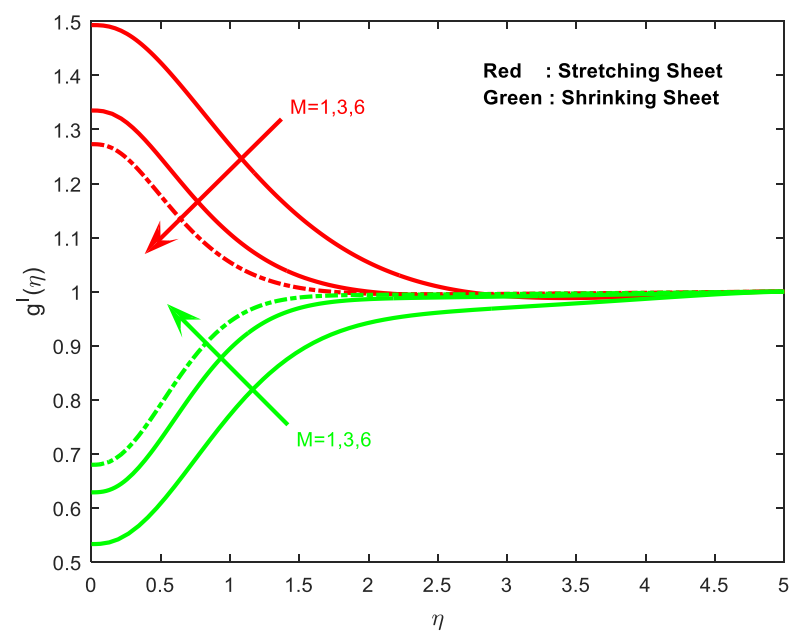

Fig. 9 Variations of $g^{\prime}(\eta)$ for different values of magnetic field parameter

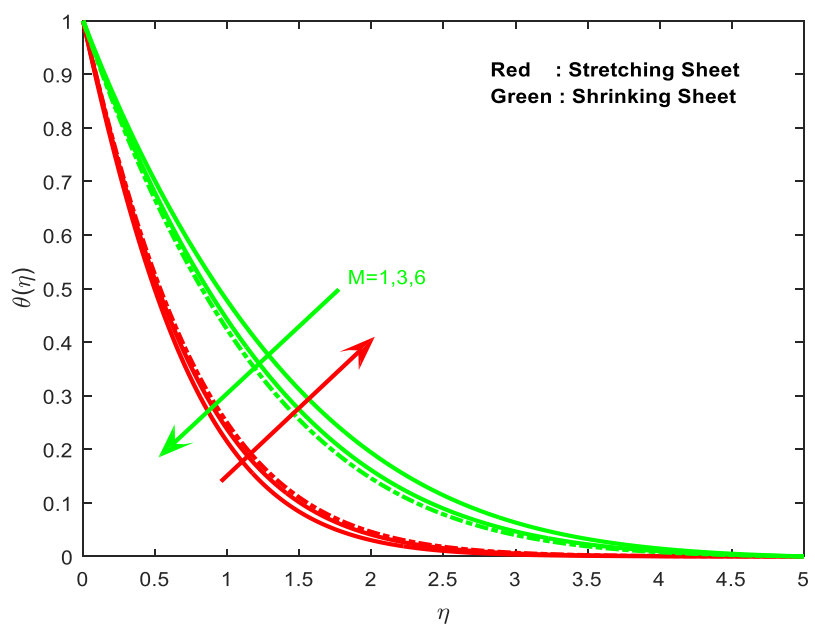

Fig. 10 Variations of $\theta(\eta)$ for different values of magnetic field parameter

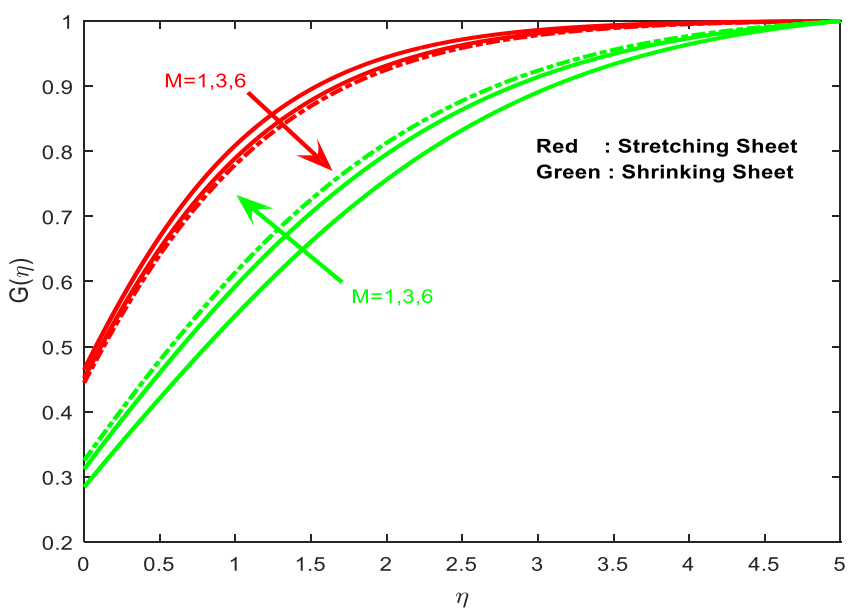

Fig. 11 Variations of $G(\eta)$ for different values of magnetic field parameter 


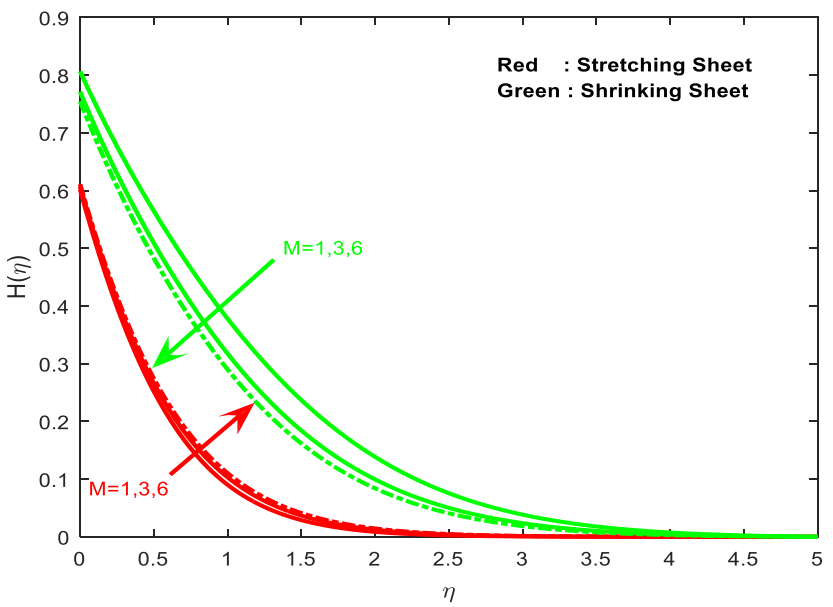

Fig. 12 Variations of $H(\eta)$ for different values of magnetic field parameter

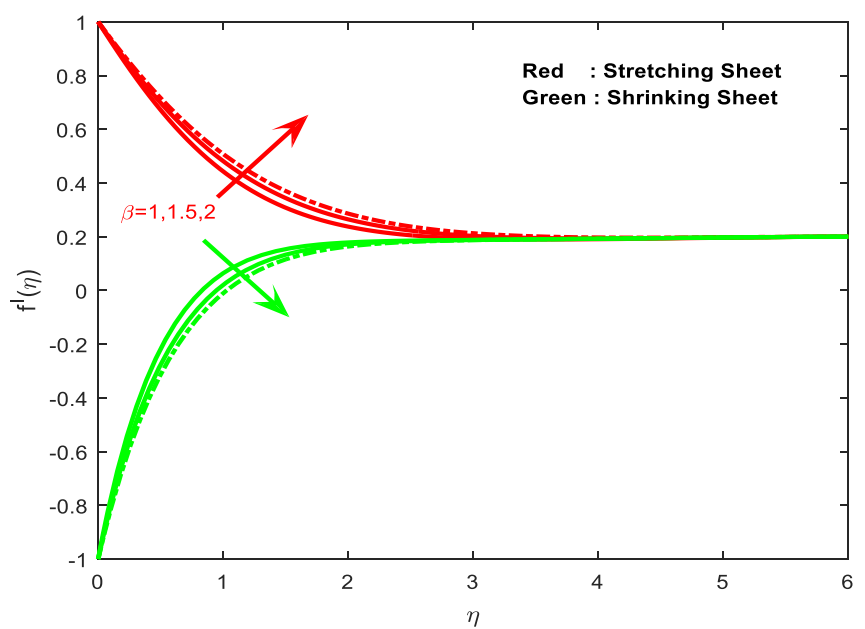

Fig .13 Variations of $f^{\prime}(\eta)$ for different values of Deborah number

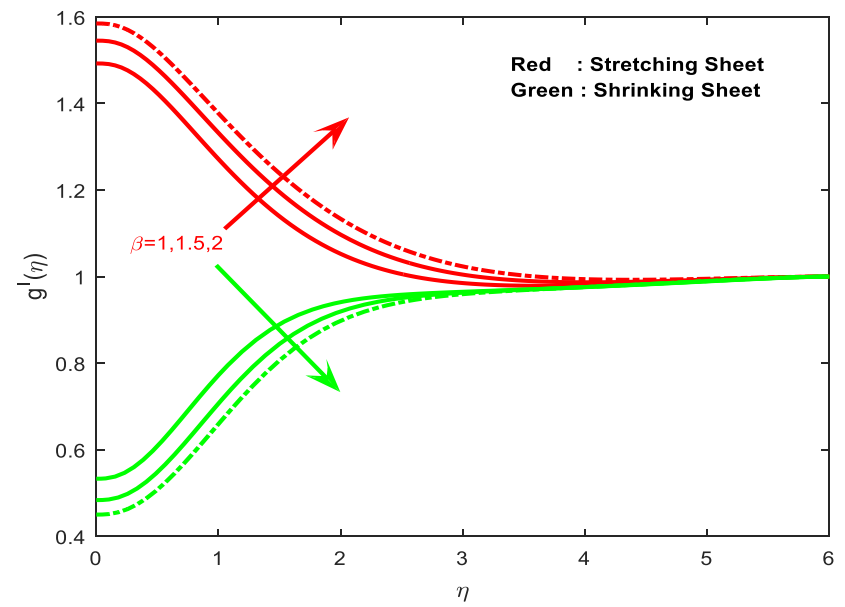

Fig. 14 Variations of $g^{\prime}(\eta)$ for different values of Deborah number

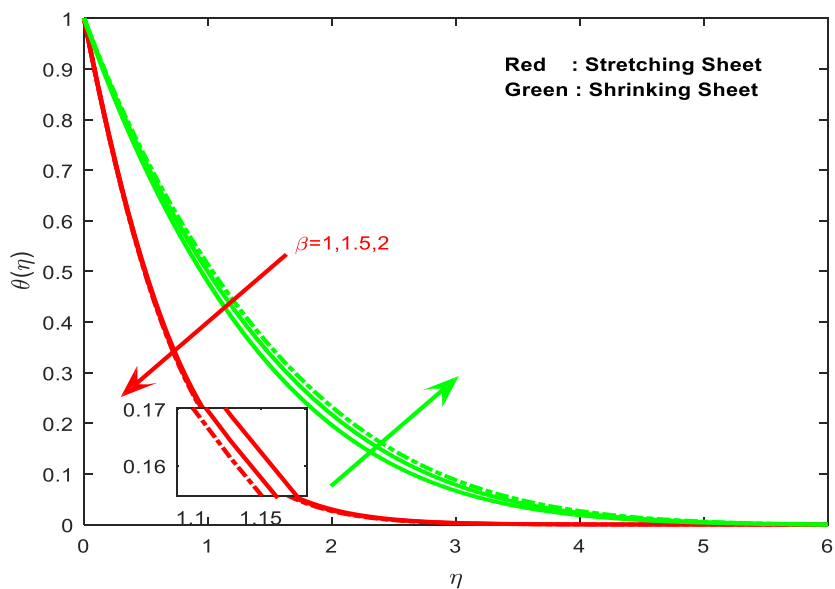

Fig. 15 Temperature profiles for different values of Deborah number

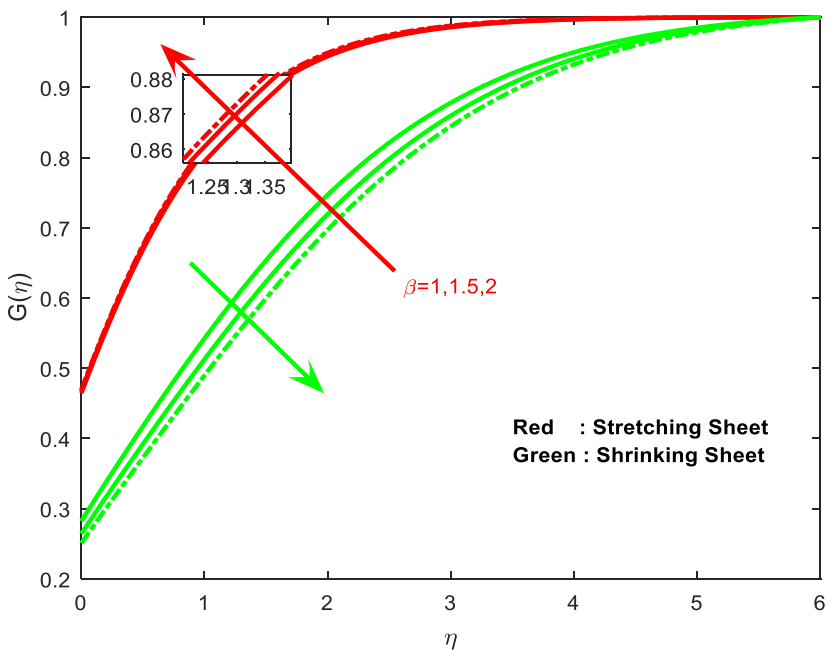

Fig. 16 Variations of $G(\eta)$ for different values of Deborah number

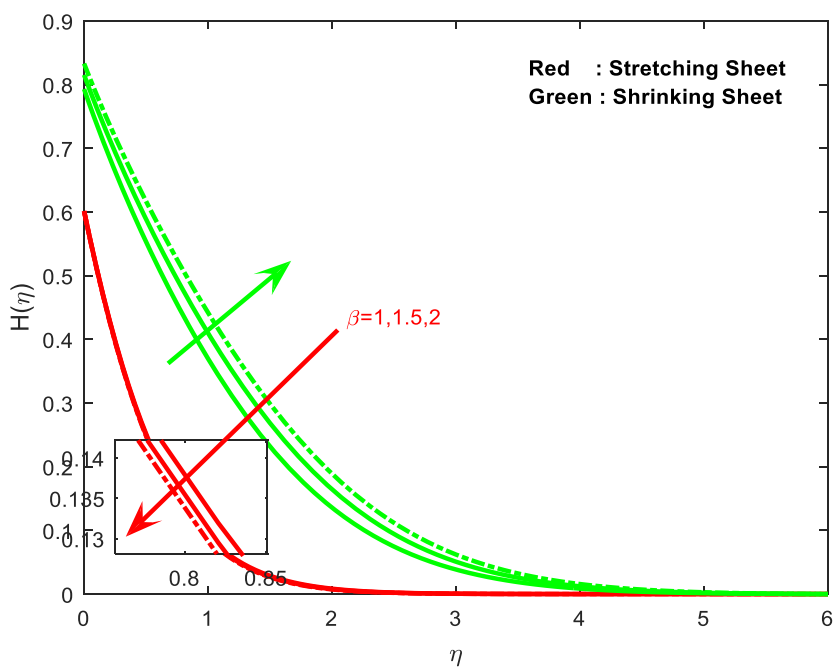

Fig. 17 Variations of $H(\eta)$ for different values of Deborah number 


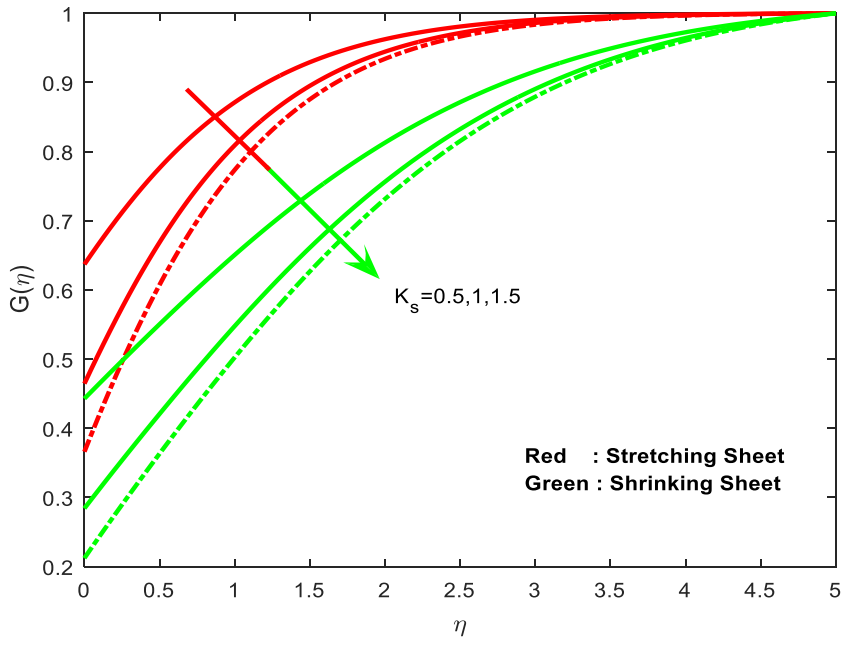

Fig. 18 Variations of $G(\eta)$ for different values of strength of heterogeneous reaction parameter

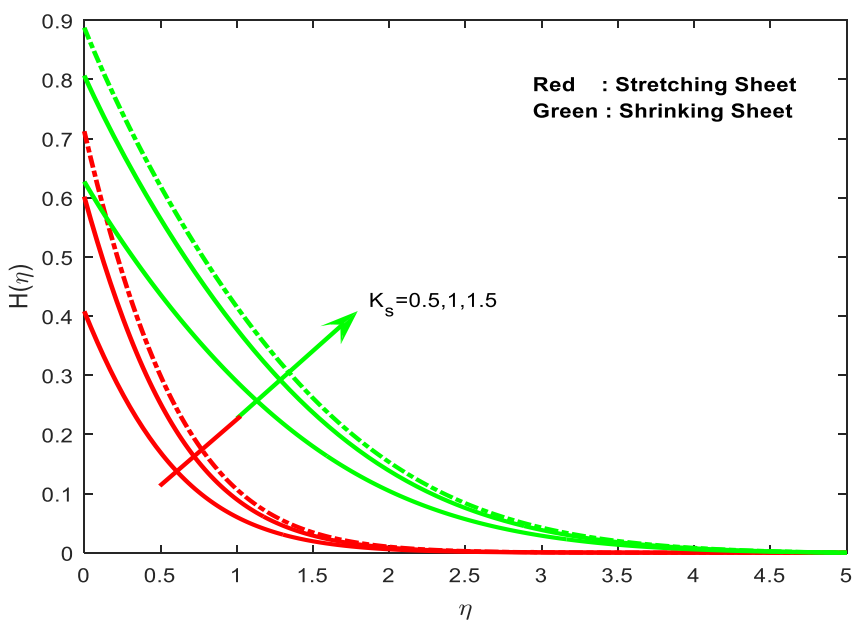

Fig. 19 Variation of $H(\eta)$ for different values of strength of heterogeneous reaction parameter

\section{CONCLUSIONS}

In this paper we considered the induced magnetic field effect on Jeffrey fluid flow past a stretching/shrinking sheet in the presence of nonlinear thermal radiation and homogeneous-heterogeneous reactions. The arising sets of governing nonlinear coupled partial differential equations are transformed into a set of nonlinear coupled ordinary differential equations using self-similarity transformations, which are then solved numerically. The conclusions are as follows:

- The heat transfer performance on the flow over a stretching sheet is comparatively better than the heat transfer performance on the flow over a shrinking sheet.

- A raise in the suction parameter encourages the heat transfer rate for both stretching and shrinking cases. But it showed a mixed response in the friction coefficient.

- The nonlinear thermal radiation enhances the thermal boundary layer thickness and decreases the rate of heat transfer.

- An increase in the magnetic field parameter decreases the skin friction coefficient and the heat transfer rate in stretching case.
- Homogeneous and heterogeneous reaction parameters are helpful to control the concentration profiles of the flow.

- Dual solutions exits only for certain range of stretching/shrinking parameter.

\section{NOMENCLATURE:}

$u, v \quad$ :Velocity components in $x$ and $y$ directions

$B_{1}, B_{2} \quad$ :Magnetic components in $x$ and $y$ directions

$B_{0} \quad$ :Magnetic component in free stream flow

$x \quad$ : Distance along the surface

$y \quad$ : Distance normal to the surface

$c_{p} \quad:$ Specific heat capacity at constant pressure

$f \quad$ :Dimensionless velocity

$u_{e} \quad$ : Free stream velocity

$T \quad$ : Temperature of the fluid

$C_{f} \quad:$ Skin friction coefficient

$\mathrm{Nu} \quad$ : Local Nusselt number

$\operatorname{Re}_{x} \quad$ : Local Reynolds number

Pr $\quad$ : Prandtl number

$\Lambda \quad$ : Porosity constant

$\lambda_{1}, \lambda_{2} \quad$ : Relaxation and retardation times

$g \quad$ : Dimensionless velocity

a,d : Positive constants

$\beta \quad$ : Deborah number

$R \quad$ : Radiation parameter

$k \quad$ : Thermal conductivity

$K \quad$ : Strength of homogeneous reaction

$K_{s} \quad$ :Strength of heterogeneous reaction

$\delta \quad$ : Ratio of diffusion coefficients

\section{Greek Symbols:}

$a, b \quad$ : Rate constants

$\eta \quad:$ Similarity variable

$\sigma \quad$ : Electrical conductivity

$\sigma^{*} \quad:$ Stefan-Boltzmann constant

$k^{*} \quad:$ Mean absorption coefficient

$\theta \quad:$ Dimensionless temperature

$\rho \quad:$ Density

$\lambda \quad$ : Stretching/shrinking parameter

$\varepsilon \quad:$ Reciprocal magnetic Prandtl number

$\mu_{e} \quad$ : Magnetic Permeability

$\mu \quad$ : Dynamic viscosity

$v \quad$ : Kinematic viscosity

$D_{A}, D_{B}$ : Diffusion coefficients

$\alpha \quad:$ Thermal diffusivity

$d / c \quad:$ stretching ratio parameter

Subscripts:

$f \quad$ : Fluid

$w \quad$ : Condition at the wall

$\infty \quad$ : Condition at the free stream

\section{REFERENCES}

Abbas, Z., Sheikh, M., And Pop, I., 2015, "Stagnation-point Flow of a Hydro-magnetic Viscous Fluid over Stretching/Shrinking Sheet with Generalized Slip Condition in the Presence of Homogeneousheterogeneous Reactions," J. Taiwan Institute of Chemical Eng. 55, 69-75.

http://dx.doi.org/10.1016/j.jtice.2015.04.001 
Ali, F. M., Nazar, R., Arfin, N. M., Pop, I., 2011, "MHD Stagnation Point Flow and Heat Transfer towards Stretching Sheet with Induced Magnetic Field," Appl. Math. Mod-ed. 32(4), 409-418.

Awais, M., Hayat, T., Nawaz, M., Alsaedi, A., 2015, "Newtonian Heating, Thermal-diffusion And Diffusion-thermo Effects in an Axisymmetric Flow of a Jeffrey Fluid over a Stretching Surface," Brazilian J. Chemical Eng. 32(02), 555-561

http://dx.doi.org/10.1590/0104-6632.20150322s00001918

Abbasi, F. M., Shehzad, S. A., Hayat, T., Alsaedi, A., Obid, M. A., 2015, "influence of Heat and Mass Flux Conditions In Hydro-magnetic Flow of Jeffrey Nanofluid," AIP Advances, 5, 037111. http://dx.doi.org/10.1063/1.4914549

Chaudhary, M. A., Merkin, J. H., 1995, A Simple Isothermal Model for Homogeneous-heterogeneous Reactions in Boundary Layer Flow: I. Equal Diffusivities" Fluid Dynamics Res. 16, 311-333. http://dx.doi.org/10.1016/0169-5983(95)00015-6

Cheng, W. T., Huang, C. N., 2004, "Unsteady Flow and Heat Transfer on an Accelerating Surface with Blowing or Suction in the Absence of and Presence of Heat Source or Sink", Chemical Engineering Science. 59, 771-780.

http://dx.doi.org/10.1016/j.ces.2003.11.019

Dabiri, S., Tryggvason, G., 2015, "Heat Transfer in Turbulent Bubbly Flow in Vertical Channels", Chemical Engineering Science 122, 106113

http://dx.doi.org/10.1016/j.ces.2014.09.006

Hayat, T., Awais, M., Obaidat, S., 2012,"Three Dimensional Flow of a Jeffrey Fluid over a Linearly Stretching Sheet," Commun. Non-linear Sci. Numer. Simulat. 17, 699-707.

http://dx.doi.org/10.1016/j.cnsns.2011.05.042

Hayat, T., Shehzad, S. A., Qasim, M., Obaidat, S., 2012, "radiative Flow of Jeffery Fluid in a Porous Medium with Power Law Heat Flux and Heat Source," Nuclear Engineering and Design, 243, 15-19 http://dx.doi.org/10.1016/j.nucengdes.2011.11.005

Hayat, T., Abbas, Z., Sajid, M., 2007, "On the Analytic Solution of Magneto Hydrodynamic Flow of a Second Grade Fluid over a Shrinking Sheet," J. Applied Fluid Mechanics, 74, 1165-1171. http://dx.doi.org/10.1115/1.2723820

Ishak, A., Nazar, R., Pop, I., 2006, "Mixed Convection Boundary Layers In the Stagnation-point Flow towards a Vertical Stretching Sheet," Meccanica, 41, 509-518.

http://dx.doi.org/10.1007/s11012-006-0009-4

Mallikarjuna, B., Rashad, A.m., Chamkha, A. J., Hariprasad Raju, S., 2015, "Chemical Reaction Effects On MHD Convective Heat And Mass Transfer Flow Past A Rotating Vertical Cone Embedded In A Variable Porosity Regime," Africa Mathematica, 27(3), 645-665. http://dx.doi.org/10.1007/s13370-015-0372-1

Mahapatra, T. R., Guptha, A. S., 2002, "heat Transfer in Stagnationpoint Flow towards a Stretching Sheet," Heat and Mass Transfer, 38 517-521

http://dx.doi.org/10.1007/s002310100215
Mohan Krishna, P., Sandeep, N., Sugunamma, V., 2015, "Effects of Radiation and Chemical Reaction on MHD Convective Flow over a Permeable Stretching Surface with Suction and Heat Generation," Walailak J. Sci. Tech., 12(9), 831-847

Mohd Rohni, A., Ahmad, S., Pop, I., 2012, "Flow and Heat Transfer over an Unsteady Shrinking Sheet with Suction in Nano Fluids," Int. J. Heat. Mass Transfer, 55(7-8), 1888-1895.

http://dx.doi.org/10.1016/j.ijheatmasstransfer.2011.11.042

Nazar, R., Amin, N., Filip, D., Pop, I., 2004, "Unsteady Boundary Layer Flow in the Region of the Stagnation Point on a Stretching Sheet," Int. J. Eng. Sci., 42, 1241-1253.

http://dx.doi.org/10.1016/j.ijengsci.2003.12.002

Qasim, M., 2013, "Heat and Mass Transfer in a Jeffrey Fluid over a Stretching Sheet with Heat Source/sink," Alexandria Engineering J. 52, 571-575 http://dx.doi.org/10.1016/j.aej.2013.08.004

Raju, C.s. K., Sandeep, N., Sulochana, C., Sugunamma, V., Jayachandra Babu, M., 2015, "Radiation, Inclined Magnetic Field and Cross-diffusion Effects on Flow over a Stretching Surface," Journal of Nigerian Mathematical Society, 34, 169-180 http://dx.doi.org/10.1016/j.jnnms.2015.02.003

Ramana Reddy, J. V., Raju, C. S. K., Jayachandra Babu, M., Sandeep, N., Sugunamma, V., 2015, "radiation And Soret Effects of Mhd Nanofluid Flow Over A Moving Vertical Moving Plate In Porous Medium," Chemical And Engineering Research 30, 9-23.

Sandeep, N., Raju, C. S. K., Sulochana, C., Sugunamma, V., 2015, "effects of Aligned Magnetic Field and Radiation on the Flow of Ferrofluids Over a Flat Plate with Non-uniform Heat Source/sink," Int. J. Sci. Eng. 8(2), 151-158.

Sheikholeslami, M., Abelman, S., 2015, "two-phase Simulation of Nanofluid Flow and Heat Transfer in an Annulus in the Presence of an Axial Magnetic Field," IEEE Transaction on Nanotechnology, 14(3), 561-569.

http://dx.doi.org/10.1109/TNANO.2015.2416318

Sulochana, C., Sandeep, N., 2015,"Dual Solutions for Radiative MHD Forced Convective Flow of a Nanofluid over a Slandering Stretching Sheet in Porous Medium," J. Naval Architecture and Marine Eng., 12, 115-124.

http://dx.doi.org/10.3329/jname.v12i2.23638

Sulochana, C., Sandeep, N., 2016,"flow And Heat Transfer Behavior of MHD Dusty Nanofluid Past a Porous Stretching/shrinking Cylinder at Different Temperatures," Journal of Applied Fluid Mechanics, 9(2), 543-553.

Turkyilmazoglu M., Pop I., 2013,"Exact Analytical Solutions for the Flow and Heat Transfer near the Stagnation Point on a Stretching/shrinking Sheet in a Jeffrey Fluid," Int. J. Heat And Mass Transfer, 57, 82-88. http://dx.doi.org/10.1016/j.ijheatmasstransfer.2012.10.006

Yacob, N., Ishak, A., 2012,"Micropolar Fluid Flow over a Shrinking Sheet," Meccanica, 47, 293-299.

http://dx.doi.org/10.1007/s11012-011-9439-8 